\title{
Efficient Cluster based Adaptive Routing for Wireless Sensor Network
}

\author{
Deeksha Verma \\ Dept. of Computer Applications \\ Kanpur Institute of Technology, Kanpur, India
}

\author{
Arun Kumar Tripathi \\ Associate Professor \\ Krishna Institute of Engineering and Technology, \\ Ghaziabad, India
}

\begin{abstract}
Wireless Sensor network consist distributed sensors to monitor external conditions and to cooperatively pass their data through the network to a base station. Clustering is an important mechanism in wireless sensor networks for obtaining scalability and better network performance.
\end{abstract}

The mail objective of this paper is to provide distributed algorithm for clustering. This paper proposed an algorithm of LEACH protocol which is intended to extend the life of the network. The proposed protocol basically has set-up and steady-state phases for each round in the protocol. Clustering through distributed method is being developed for dealing with the issues like cluster formation and network performance.

\section{General Terms}

LEACH Protocol, Network Performance

\section{Keywords}

Base station, Set-up phase, Steady-state phase, Cluster-head, Clustering algorithm, Static- clustering algorithm.

\section{INTRODUCTION}

Wireless Sensor Network (WSN) consist of a number of small battery- powered devices, are frequently obtained various sort of useful data from surrounding. These devices sense physical properties and transmit the data to a base station (BS) for further analysis and processing. WSN have been effectively deployed in tactical combat situation, habitat monitoring, home security and so on [1-5]. Since WSN consist of many sensors with limited energy, an energy-efficient network protocol is an important consideration in WSN applications.

WSN comprises two classes of node, namely primary and secondary nodes. Primary node equipped with sensor and radio system and Secondary node are simply the forwarding nodes which have a radio alone to act as bridge nodes.

Clustering is the grouping of similar objects and a clustering of a set is partition of its elements that is chooses to minimize some measure of dissimilarities [6]. Clustering algorithm are often useful in applications in various fields such as visualization, pattern recognition, learning theory, computer graphics, neural networks, artificial intelligence and statistics. A practical application [7] of clustering includes pattern recognition under unsupervised learning, time series analysis, text mining and navigation.

A group of nodes form a cluster and the local interaction between clusters member are controlled through a cluster head (CH) [8]. Cluster members [9] communicate with the cluster head and collected data are aggregated and fussed by the cluster head. The cluster head can also form another layer of clusters among themselves before reaching the sink. Clustering algorithms limits the communication in a local domain and transmit only necessary information to the rest of the network through the gateway nodes.

A popular centralized as well as distributed deterministic partitional clustering approach is offered by algorithm, which features are simple, highly reliable and fast-convergent iterations and re-clustering during failure states [10].

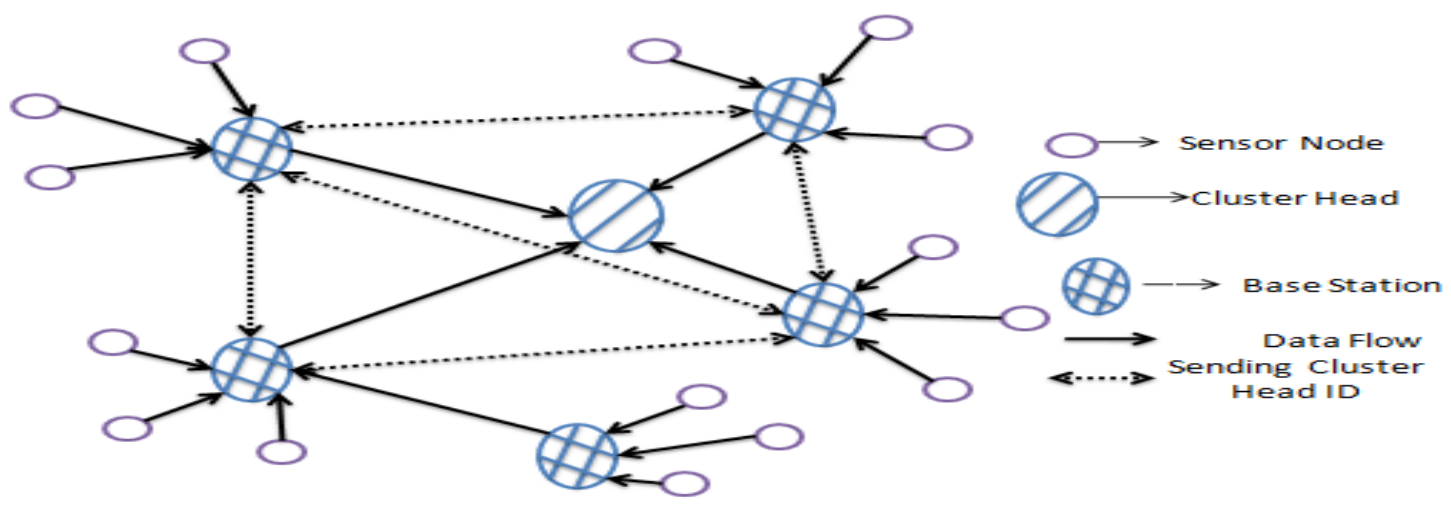

Fig 1: Data Communication in cluster network

\section{RELATED WORK}

Clustering is the method by which sensor nodes in a network organize themselves into hierarchical structure. By this, sensor nodes can use the network resource such as radio resource, battery power more efficiently. Cluster formation is based on energy remaining of sensor nodes and sensor proximity to cluster head. The role of cluster head is to forward these data and its own data to the base station after performing data aggregation and fusion. LEACH is one of the first hierarchical routing protocols for WSN. The idea 
proposed in LEACH has inspired by many other hierarchical routing protocol $[11,12]$.

In secure data collection, mobile data collector is used to collect the data from the non-cluster head nodes. More or less resembles the older version of using gateways between clusters [13] have addressed the hot spot problem effectively in WSN clustering.

\section{PROPOSED WORK}

LEACH protocol is a typical representation of hierarchical routing protocols. IT is self-adaptive and self- organized. The proposed protocol uses round as unit, each round is made up of cluster set-up stage and steady-state stage, for the purpose of reducing unnecessary energy cost, steady-state stage must be much longer than the set-up stage. The process of formation of cluster is shown in fig.2.

This protocol is suitable for the WSN under the following assumption:

* All sensor nodes are identical and charged with the same amount of initial energy and all nodes consumes energy at same rate.

* Every node can directly communicate with every other node, including the sink node.

* Sink node is fixed and away from WSN. Thus we can ignore the energy consumed by the sink node.
We assume that it always has sufficient energy to operate.

* Every node has data to transfer in every time frame. Data transferred by nodes are related and can be fused.

* Sensor nodes are static.

At the stage of cluster forming, a node randomly picks a number between 0 to 1 , compared this number to the threshold values $t(n)$, if the number is less than $t(n)$ then it become cluster head in this round, else it become common node. Threshold $\mathrm{t}(\mathrm{n})$ is determine by the following-

$$
\mathrm{t}(\mathrm{n})=\mathrm{p} / 1-\mathrm{p} *(\mathrm{r} \bmod 1 / \mathrm{p})----------------------(1)
$$

Where, $\quad \mathrm{p}=$ cluster head probability

$$
\begin{aligned}
& t(n)=\text { threshold value } \\
& r=\text { random number between } 0 \text { and } 1
\end{aligned}
$$

We introduce an algorithm- based variant of LEACH to determine the optimal value of $p$ for various base station placements. This optimization procedure is performed only once, before the set-up phase of the first round. Pseudo code of the proposed protocol is described by set-up phase and steady- state phase.

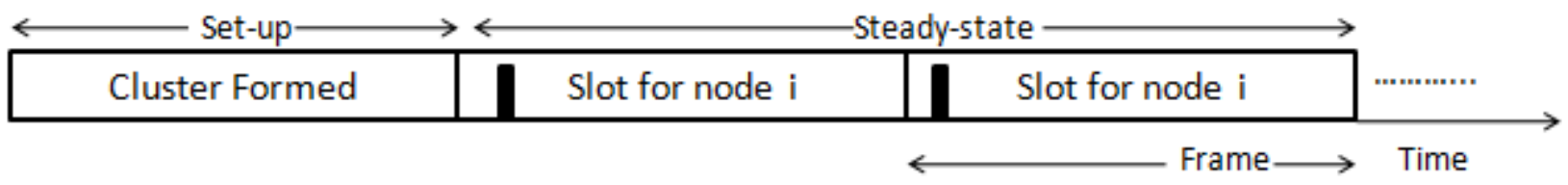

\section{Fig 2: LEACH Protocol Process}

\subsection{Set-up Phase}

The cluster formation algorithm should be designed such that nodes are cluster-heads at the same number of time, by assuming all the nodes start with the same amount of energy [14]. The cluster-head node should be spread throughout the network, as this will minimize the distance according to noncluster- head nodes to send their data. A sensor node has chosen a random number $\mathrm{r}$, between 0 and 1 . If this random number is less than a threshold value $\mathrm{t}(\mathrm{n})$, then node becomes a cluster-head for the current round. Threshold value is calculated by equation (1) that incorporates the desired percentage to become a cluster-head for the current round and the set of nodes that have not been selected as a cluster-head for the last $(1 / \mathrm{p})$ rounds.

After the selection of cluster-head, it broadcast the message (MSG). This message is small which contains node ID and a header that distinguished this message as an announcement message. The cluster head act as a local control center to coordinate the data transmissions in their cluster [15]. This ensures that there are no collisions among data messages and also allows the radio components of each non-cluster head node to be turned off at all time except during their transmit time, that minimize the energy dissipated by the individual $[16,17]$.

\subsection{Steady-state Phase}

The steady-state phase is broken in to frames where nodes send their data to the cluster-head at most onetime per frame during their allocated transmission frame. The number of per cluster is highly variable and the amounts of data for each node can send to the cluster head varies depend on the number of nodes in the cluster. The cluster head must keep its receiver to receive all the data from the nodes in the cluster. When cluster head received all the data, it can operate on the data and then the resustaned data are sent from the cluster head to the base station.

Algorithm for the Set-up phase and Steady-state phase in wireless sensor network is described below-

\section{SET-UP PHASE}

1. Specify the probability $p$, number of nodes $n$;

2. $\mathrm{E}(\mathrm{n})=\mathrm{E}_{0}, \quad \mathrm{n}=0,1,2,3 \ldots \ldots \ldots \ldots \ldots$;

3. do $\{$ //repeat for $r$ rounds

4. $\mathrm{r} \leftarrow \operatorname{random}(0,1)$;

5. if $(\mathrm{E}(\mathrm{n})>0$ and $\mathrm{r} \bmod (1 / \mathrm{p}) \neq 0)$

6. Then, $\quad t(n)=p / 1-p^{*}(r \bmod 1 / p)$;

7. $\quad$ If $(\mathrm{r}<\mathrm{t}(\mathrm{n}))$

8. $\quad \mathrm{CH}\{\mathrm{n}\}=$ TRUE; // node $\mathrm{n}$ be a $\mathrm{CH}$

9. else

10. $\mathrm{CH}\{\mathrm{n}\}=$ FALSE; // node $\mathrm{n}$ not be a $\mathrm{CH}$

11. endif

12. endif 

13. if $(\mathrm{CH}\{\mathrm{n}\}=$ TRUE $)$
14. then $\mathrm{BC}(\mathrm{MSG}) \leftarrow$ broadcast advertisement message;
15. Join(IDi); // non cluster head node I join into closest $\mathrm{CH}$
16. Cluster(c); // form a cluster $\mathrm{c}$
17. Endif

STEADY-STATE PHASE

1. If $(\mathrm{CH}(\mathrm{n})=\mathrm{TRUE})$ then

2. Receive(IDi, DataPCK); // receive data from members

3. Aggregate(IDi, DataPCK);// aggregate received data

4. TransToBS(IDi, DataPCK); // transmit received data

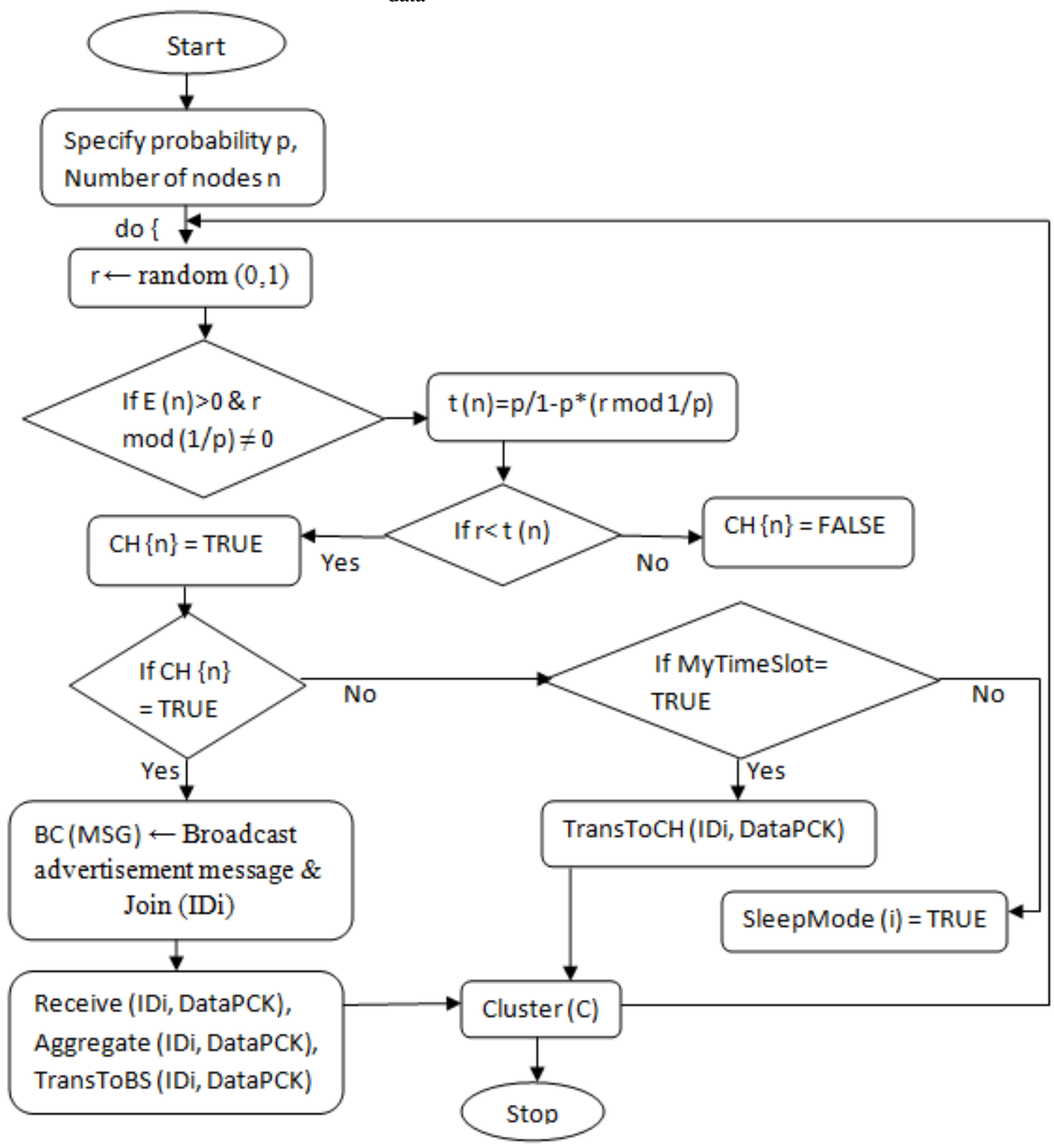

Fig 3: Data Flow Diagram of LEACH Protocol

\section{CONCLUISION}

This work proposed a adoptive clustering protocol to determine the optimal threshold probability for cluster
5. Else

6. if(MyTimeSlot=TRUE) then

7. TransToCH(IDi, DataPCK); // transmit

8. else

sensed data

9. SleepMode(i)=TRUE;// node I at a sleep

10. endif

11. endif

12. \} //one round is completed

The above algorithm can be easily understood with the help of data flow diagram shown in figure 3 . 
cluster-head or not. Our approach uses a SET-UP PHASE for the first round to gather information about node status, IDs and location and send it to the base station, which determine the optimal probability to use in the cluster-head selection. The use of the optimal probability yields optimal energyefficient clustering.

\section{REFERENCES}

[1] J. Akyildiz, W.Su, Y.Sankarasubramaniam and E.Cayirci, "A Survey on Sensor Networks," IEEE communication magazine, vol.40, no.8, Aug.2002, pp.102-104

[2] K.Akkaya and M.Younis, "A Survey on routing protocol for wireless Sensor Networks," AdHoc Networks, vol.3, May.2005, pp.325-349.

[3] A.A.Abbasi,and M.Younis, "A Survey on Clustering Algorithms for Wireless Sensor Networks," Computer Communications, vol.30, Oct.2007, pp.2826-2842.Tavel, P. 2007 Modeling and Simulation Design. AK Peters Ltd.

[4] A.MAnwaring, J.Polastre, R.Szewczyk, D.Culler and J.Anderson, "Wireless Sensor Network for habitat monitoring," in ACM WSNA'02, Atlanta, Gorgia, Sept.2002, pp.88-97.

[5] S.Zhu, W.Wang and C.V.Ravishankar, "PERT: A New Power Efficient Real-Time Packet delivery scheme for Sensor Networks," International Journal of Sensor Networks, vol.3, issue.4, Jun.2008, pp.1713-1723.

[6] S.Bandyopadhyay, E.J.Coyle, "An Energy Efficient Hierarchal clustering Algorithm for Wireless Sensor Network," IEEE INFOCOM, vol.3, 2003, pp.1713-1723.

[7] Manasi.N. Jashi, "Parallel K-means Algorithm on Distributed memory Multiprocessors," Spring.2003.

[8] W.Heinzelman, A.Chandrakasan and H.Balakrishnan, "Energy Efficient Communication Protocols for Wireless
Microsensor Networks" Proceeding of the Hawaii International Conference on Systems Sciences, Jan.2000.

[9] O.Younis, M.Krunz, S.Ramasubramanian, "Node Clustering in Wireless Sensor Network: Recent Development and Deployment Challenges," IEEE Network, vol.20, issue.3, May.2006, pp.20-25.

[10] S.P.Lioyd, "Least-square quantization in PCM," IEEE Trans.Inf.Theory, vol.IT-28, no.2, pp.129-137, Mar.1982.

[11] A.Manjeshwar, D.P.Agrawal, APTEEN, "A Protocol for enhanced efficiency in Wireless Sensor Networks," Proceeding of the $1^{\text {st }}$ international Workshop on Parallel and Distributed Computing issue in Wireless Networks and Mobile Computing, San Fransisco, CA, April.2001.

[12] A.Manjeshwar, D.P.Agrawal, APTEEN, “A Hybrid Protocol for efficient routing and comprehensiveinformation retrieval in Wireless Sensor Networks," Proceeding of $2^{\text {nd }}$ International Workshop on Parallel and Distributed Computing issues in Wireless Networks and Mobile Computing, Ft.Lauderdale, FL, April.2002.

[13] Y.Liu, N.Xiong, Y.Zhao, A.V.Vasilakos, J.Gao and Y.Jia, "Multi-Layer Clustering routing Algorithmfor Wireless Vehicular Sensor Network," IET Commun., 2010, vol.4.Iss.7, pp.810-816.

[14] M.J.Handy, M.Haase, D.Timmermann, "Low Energy Adaptive Clustering Hierarchy with Deterministic Cluster-head Selection," IEEE MWCN, 2002.

[15] W.B.Heinzelman, "An Application Specific Protocol for Wireless Microsensor Networks," IEEE TRANSACTION WIRELESS COMMUNICATION, Oct.2001.

[16] Zhang, Yu-quan, Wei, Lei, "Improving the LEACH protocol for Wireless Sensor Networks," Wireless Sensor Network, 2010.IET-WSN.IET International Conference. 